\title{
La podredumbre radical del alcornoque y el acebuche. Efecto del contenido hídrico del suelo y control de la enfermedad mediante inductores de resistencia
}

\author{
Cork oak and wild olive root rot. Effect of soil water content \\ and disease control through resistance inducers
}

\author{
González, M. ${ }^{*}$
}

${ }^{1}$ Universidad de Córdoba, Departamento de Agronomía,

Ctra Madrid-Cádiz 396, 14014, Córdoba, España 


\section{Resumen}

El acebuche y el alcornoque son dos de las especies más importantes de los ecosistemas mediterráneos. Sin embargo, en los últimos años, ambas especies están siendo gravemente afectadas por podredumbres radicales, decaimiento y muerte del arbolado, que ponen en grave peligro su conservación. Esta Tesis Doctoral ha permitido determinar la etiología de las podredumbres radicales del acebuche, incluyendo la primera descripción de Phytophthora oleae como patógeno radical de Olea spp. Además, ha permitido esclarecer algunos aspectos de la epidemiología de la podredumbre radical del alcornoque causada por P. cinnamomi y Pythium spiculum, como la influencia del estrés hídrico y del contenido de humedad del suelo en el desarrollo de la enfermedad. Asimismo, se ha demostrado, aportando evidencia experimental contrastada, el efecto preventivo y curativo de los fosfonatos para evitar la destrucción de los ecosistemas mediterráneos dominados por el alcornoque.

Palabras clave: Phytophthora, oomicetos, Quercus suber, Olea europea.

\section{Summary}

Wild olive and cork oak are keystone tree species of high ecological value for Mediterranean forests. Both species are suffering root rots, decline, and death, which seriously threaten their future. This PhD thesis state the etiology of the root rots affecting wild olives, including the first report of the new pathogen $P$. oleae causing root disease on Olea spp. Furthermore, the influence of previous drought and soil water content in the epidemiology of cork oak root disease caused by P. cinnamomi and Pythium spiculum has been clarified. In addition, has provided statistical evidence of the preventive and therapeutic effectiveness of systemic phosphonates to avoid destruction of Mediterranean ecosystems based on oak woodlands.

Keywords: Phytophthora, oomycetes, Quercus suber, Olea europea. 


\section{Introducción}

El alcornoque (Quercus suber L.) y el acebuche (Olea europaea subsp. europaea var. sylvestris) constituyen dos de los elementos clave de los bosques mediterráneos (Costa et al., 1997). Estos ecosistemas son especialmente proclives a sufrir procesos de decaimiento provocados, entre otras causas, por la incidencia de enfermedades y plagas (Vitousek 1994). En este contexto, uno de los mayores problemas a los que se enfrentan los ecosistemas forestales mediterráneos son las enfermedades radicales causadas por oomicetos (Liebhold et al., 2017; Sena et al., 2018). Estos microorganismos producen una destrucción masiva de raíces absorbentes de los árboles que infectan, reduciendo su capacidad de absorción de agua y nutrientes, y generando en la parte aérea una sintomatología muy inespecífica: amarillez y/o marchitez foliar, defoliación y muerte regresiva de brotes y ramas (Erwin y Ribeiro 1996). El acebuche, en general, es una especie forestal poco susceptible a la incidencia de enfermedades. Sin embargo, en uno de los acebuchales más importantes de Europa (Smits et al., 2005), se observó en el año 2009 la sintomatología típica de una podredumbre radical. Por ello, se planteó la necesidad de determinar la etiología de esta enfermedad, que puede poner en peligro la conservación de un acebuchal de alto valor ecológico.

Por otra parte, la podredumbre radical causada por Phytophthora cinnamomi y Pythium spiculum se ha convertido en la principal causa de muerte del alcornoque en el suroeste de la Península Ibérica (Sánchez et al., 2006; Romero et al., 2007). Phytophthora cinnamomi es el único microorganismo incluido en las 100 especies exóticas invasoras más dañinas del mundo (Lowe et al., 2000). Existen numerosos estudios sobre diferentes aspectos epidemiológicos de la enfermedad radical que causa en los Quercus mediterráneos (Sánchez et al., 2002; Hardham 2005; Desprez-Loustau et al., 2006; Corcobado et al., 2013). Sin embargo, quedaban por aclarar algunos aspectos relevantes, como el papel de la sequía, tantas veces citado como un factor predisponente a la infección por $P$. cinnamomi, o su capacidad de competencia con el otro oomiceto implicado, Py. spiculum, para infectar la raíz del alcornoque, tanto en las condiciones climáticas actuales, como bajo las futuras condiciones previstas por los modelos de cambio climático.

En cuanto al control de este tipo de enfermedades en especies forestales, cabe destacar el manejo integrado de la enfermedad en dehesas de encina mediante un buen manejo del ganado, drenajes (Fernández et al., 2008), selección de cultivos herbáceos no susceptibles al patógeno (Serrano et al., 2012a), fertilización cálcica (Serrano et al., 2012b), o biofumigación (Ríos et al., 2016). Sin embargo, la mayoría de estas medidas son de difícil aplicación en un medio natural no antropizado. En ecosistemas naturales, las podredumbres radicales suelen controlarse principalmente mediante la aplicación de fosfonatos, siendo un ejemplo paradigmático el control de la podredumbre radical de eucaliptos en bosques naturales australianos. Los fosfonatos son productos fitosanitarios inductores de resistencia (fitoactivadores) de carácter sistémico. Son sales de bajo peso molecular del ácido fosforoso, no tóxicas y absorbibles por la planta, capaces de desplazarse por el xilema (movimiento acrópeto) y el floema (movimiento basípeto). Por este motivo, a diferencia de otros pro- 
ductos sistémicos, los fosfonatos pueden ser aplicados al tronco mediante inyección (Hardy et al., 2001; Garbelotto et al., 2007) o pulverizarse sobre el follaje (Jackson et al., 2000; Hardy et al., 2001; Garbelotto et al., 2007; Shearer et al., 2012). Los productos basados en fosfito potásico han mostrado buenos resultados en el control preventivo de podredumbres radicales causadas por Phytophthora spp. en Quercus ilex (Fernández-Escobar et al., 1999); Quercus agrifolia (Garbelotto et al., 2009); Eucalyptus spp. y otras especies nativas australianas (Jackson et al., 2000; Hardy et al., 2001). Sin embargo, la comercialización de las sales del ácido fosforoso (fosfitos) como fertilizantes ha dado lugar a la prohibición de su uso en tanto no sean comercializados como productos fitosanitarios, según su adscripción en el Registro Único Europeo (European Pesticides database https://ec.europa.eu/food/plant/pesticides/eu-pesticidesdatabase/public/?event=homepage\&language=EN). Por este motivo, se hizo necesario testar otros fosfonatos sistémicos comercializados como fitosanitarios para su posible uso tanto en monte como en sistemas adehesados.

En base a estas premisas, los objetivos de esta Tesis Doctoral fueron:

* Determinar la etiología de la podredumbre radical que afecta al acebuche.

* Esclarecer la influencia del estrés hídrico del alcornoque como factor predisponente para la infección por Phytophthora cinnamomi.

* Determinar el grado de competencia entre $P$. cinnamomi y Py. spiculum para infectar la raíz del alcornoque en un gradiente de humedad del suelo.

* Evaluar la eficacia de inductores de resistencia (fosfonatos) en el control de la podredumbre radical del alcornoque y el acebuche en condiciones controladas.

* Evaluar la eficacia de inductores de resistencia (fosetil-aluminio) en el control de la podredumbre radical del alcornoque en campo.

\section{Etiología de la podredumbre radical del acebuche}

En los últimos años se han observado síntomas de podredumbre radical en el acebuchal de la Reserva Natural Concertada de la Dehesa de Abajo (Figura 1), un área protegida perteneciente al municipio sevillano de La Puebla del Río. Durante los años 2013, 2014 y 2015 se tomaron muestras de suelo y raíz de árboles sintomáticos y se analizaron posteriormente en el laboratorio. Se aislaron consistentemente tres especies del género Phytophthora (P. cryptogea, P. oleae y P. megasperma), junto con $P$ y. spiculum, demostrándose su patogenicidad en plantones. En muestreos previos (2009), P. megasperma fue el principal patógeno asociado a la enfermedad, mientras que en este trabajo se constató que, en 2013, P. cryptogea fue la principal especie aislada, probablemente debido a un aumento generalizado de las temperaturas, que favorecieron las infecciones radicales por parte de una especie 
más termófila, como es $P$. cyptogea. Además, desde su primera detección en 2014 , la nueva especie invasora $P$. oleae pareció estar desplazando a las especies de Phytophthora nativas, poniendo en peligro no solo la supervivencia de los acebuchales, sino que también representa un grave riesgo para el olivar.
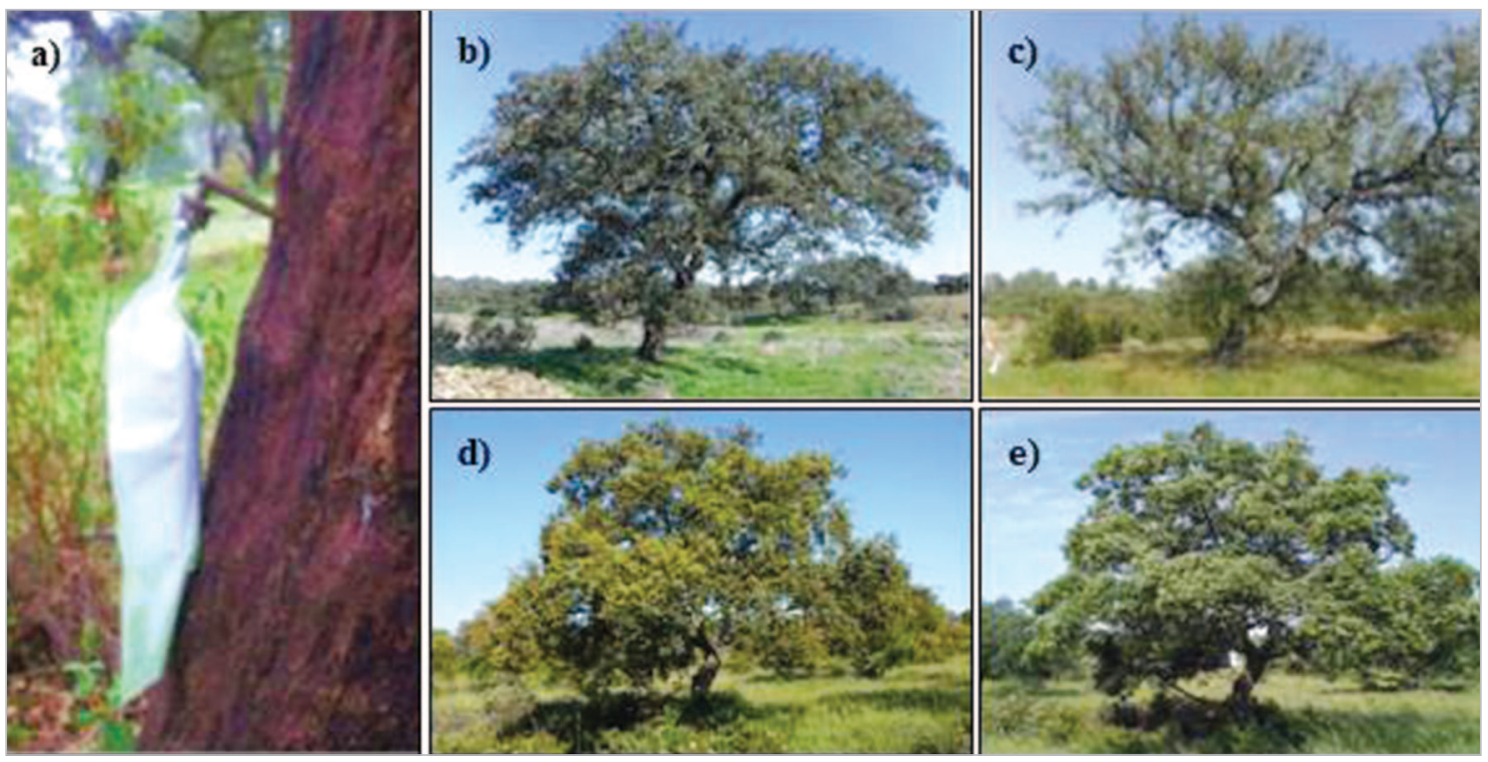

Figura 1. Vista general de un árbol sintomático (izquierda) junto a dos asintomáticos (derecha) en el acebuchal de la Reserva Natural Concertada de la Dehesa de Abajo (a). Síntomas foliares y radicales: amarillez foliar (b); defoliación severa (c); necrosis y pérdida de raíces absorbentes (d).

\section{Epidemiología de la podredumbre radical del alcornoque}

Se realizaron inoculaciones artificiales utilizando plantones de alcornoque y se evaluó la influencia de la humedad en el desarrollo de la enfermedad. Por un lado se determinó el efecto de la sequía como un factor predisponente en la infección de P. cinnamomi. Para ello, los plantones se sometieron a tres diferentes condiciones hídricas: sequía extrema, sequía moderada y riego normal. Posteriormente se inocularon con suspensiones de clamidosporas de P. cinnamomi y se mantuvieron durante cuatro semanas en condiciones óptimas para la infección. Finalmente se evaluaron los síntomas radicales y se demostró cómo este oomiceto actúa como un patógeno virulento cuando los plantones se expusieron a suelos infestados con clamidosporas, independientemente de las condiciones previas de estrés hídrico. Por lo tanto, la sequía por sí misma no puede ser considerada como un factor predisponente necesario para el desarrollo de la podredumbre radical del alcornoque.

Por otro lado, se evaluó la influencia del contenido de humedad del suelo en el nivel de competencia de los oomicetos causantes de podredumbre radical en alcornoque ( $P$. cinnamomi y Py. spiculum). Para ello, los plantones se inocularon con suspensiones de esporas de los dos patógenos y se sometieron a diferentes niveles de humedad del suelo. Se comprobó que el contenido hídrico es un factor determi- 
nante para la infección por uno u otro patógeno. Phytophthora cinnamomi fue altamente virulento en suelos con un porcentaje de humedad entre la saturación y la sequía moderada ( $\geq 25 \%$ ), causando altos niveles de necrosis radical, pero fue incapaz de infectar las raíces cuando la humedad del suelo disminuyó hasta el 12\% (sequía). Por el contrario, Py. spiculum puede infectar la raíz del alcornoque en condiciones de sequía, pero resulta menos virulento que $P$. cinnamomi en suelos húmedos, e incapaz de infectar las raíces en suelos saturados. En los alcornoques inoculados simultáneamente con ambos patógenos, los síntomas radicales fueron siempre similares a los producidos en solitario por $P$. cinnamomi en un amplio rango de humedad del suelo (9-100\%), sin ningún efecto sinérgico entre los dos patógenos. Por lo tanto, P. cinnamomi predominará como patógeno radical de Quercus en suelos saturados a moderadamente húmedos, mientras que $P y$. spiculum será el patógeno predominante solamente en ausencia de $P$. cinnamomi o en condiciones de sequía. De esta forma, los bosques de Quercus mediterráneos, habitualmente infestados por ambos patógenos, estarán en riesgo de sufrir epidemias de la enfermedad en el momento presente y también bajo las condiciones de aumento de la aridez previstas para la región mediterránea.

\section{Control de la podredumbre radical del alcornoque y del acebuche}

Por otra parte, la creciente necesidad de un manejo integral de las enfermedades que afectan a las dehesas y montes de Quercus, que además sea respetuoso con el medio ambiente, hace del uso de productos activadores de resistencia (fosfonatos) una opción atractiva. Estos productos pueden ser aplicados directamente al tronco (endoterapia), estimulando las defensas naturales del árbol frente a determinados patógenos e impidiendo la contaminación de las dehesas y alcornocales. En este contexto, se evaluó la capacidad de estos productos para inhibir in vitro el crecimiento micelial de $P$. cinnamomi y se ensayó su eficacia para prevenir la infección en plantones de alcornoque. Se demostró que elbfosfito potásico, a la dosis recomendada por el fabricante $(100 \mu \mathrm{g} / \mathrm{mL})$, inhibe totalmente el crecimiento radial de las colonias de $P$. cinnamomi, mientras que el fosetil-aluminio indujo una reducción del crecimiento del $75 \%$. Sin embargo, solamente las plantas tratadas con fosetil-aluminio mostraron unos niveles de síntomas radicales similares a los de las plantas no inoculadas.

Además, también se evaluó la eficacia del fosfito potásico y el fosetil-aluminio contra los patógenos radicales del acebuche en condiciones in vitro e in planta. El fosfito potásico redujo el crecimiento micelial de $P$. cryptogea, $P$. oleae, $P$. megasperma y $P$ y. spiculum, mientras que el fosetil-aluminio solo fue efectivo contra $P$. cryptogea y $P$. oleae. Sin embargo, cuando se ensayaron in planta, ambos fosfonatos redujeron significativamente la necrosis radical causada por todas las Phytophthora spp.

Finalmente, y debido a que el uso del fosfito potásico se prohibió en España, debido a su comercialización como fertilizante fosfórico, se ensayó la efectividad del fosetil-aluminio contra la podredumbre radical del alcornoque. Este producto es un 
fosfonato registrado en España como fungicida para su uso en cultivos agrícolas y algunos cultivos forestales. Por ello, se ensayó la eficacia del fosetil-aluminio en una dehesa de alcornoque, aplicando el producto por inyección al tronco a la dosis recomendada por el fabricante para cultivos leñosos. Los árboles se catalogaron en función de su clase de defoliación: árboles asintomáticos (0-10\% de defoliación), con defoliación leve (11-25\%) o moderada (26-50\%). Además, se tomaron muestras de raicillas absorbentes y de suelo de la rizosfera de cada árbol para el aislamiento de patógenos. Se inyectaron 30 árboles (10 por clase de defoliación) con fosetil-aluminio, y otros 30 (testigos) solamente con agua. Los alcornoques, incluyendo algunos asintomáticos, ya estaban infectados con P. cinnamomi y Py. spiculum cuando se realizó el tratamiento con fosetil-aluminio o con agua. La evaluación de la defoliación y los muestreos de raíz y suelo se repitieron dos veces al año durante 3 años. Al final del periodo de evaluación, la aplicación de fosetil-aluminio redujo significativamente la defoliación de los árboles tratados en comparación con los testigos, independientemente de la clase de defoliación inicial (Figura 2). Ade-
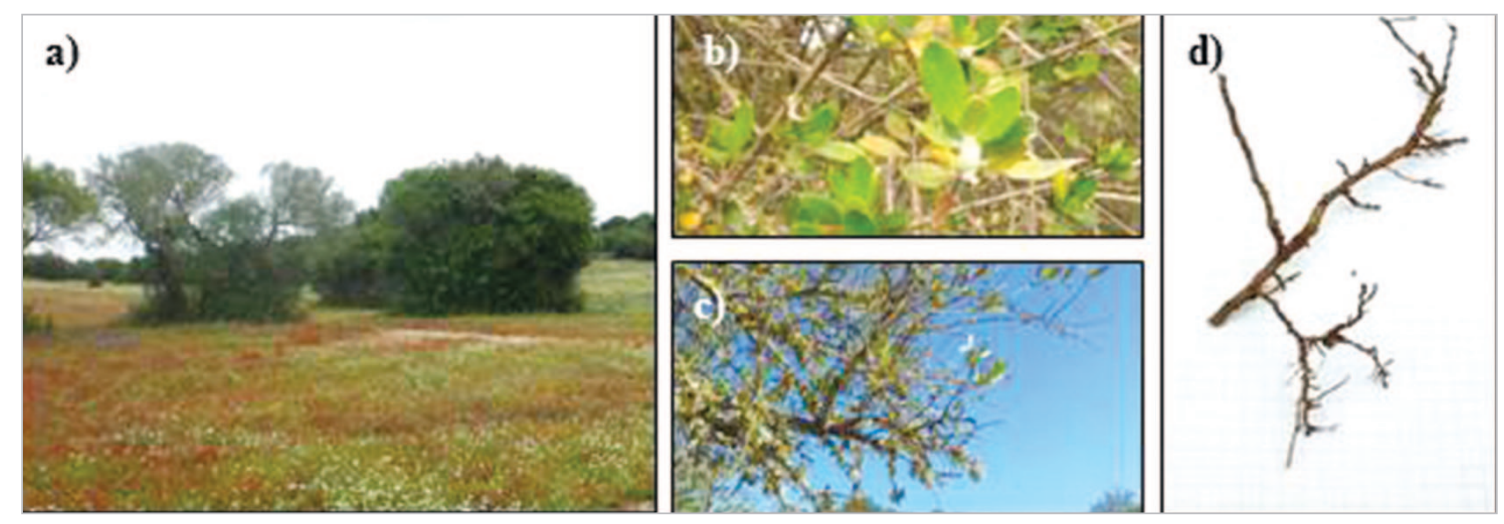

Figura 2. Efectividad del fosetil-aluminio contra la podredumbre radical del alcornoque en dehesa. Inyección al tronco de un alcornoque mediante una cápsula presurizada conteniendo fosetil-aluminio (a). Aspecto de la copa de un alcornoque testigo (tratado con agua) en el año 2014 (b) y 2017 (c). Obsérvese la pérdida de follaje. Aspecto de la copa de un alcornoque tratado con fosetil-aluminio en el año 2014 (d) y 2017 (e). Véase cómo el árbol permanece con un alto porcentaje de cobertura foliar.

más, se observó una mejora de la cobertura foliar en los árboles tratados, lo que sugiere un efecto terapéutico del fosetil-aluminio sobre las infecciones preexistentes, que se sumaría a su efecto preventivo contra las nuevas infecciones. Por otro lado, las variaciones en la densidad de inóculo en el suelo o en la presencia de P. cinnnamomi y Py. spiculum en las raíces a lo largo del tiempo de evaluación, no dependieron significativamente del tratamiento aplicado. Por lo tanto, la aplicación de inyecciones al tronco de fosetil-aluminio debe considerarse como una medida efectiva en el manejo integrado de esta grave enfermedad del alcornoque, incluso en áreas naturales protegidas. 


\section{Bibliografía}

Corcobado, T.; Cubera, E.; Moreno, G.; Solla, A.; 2013. Quercus ilex forests are influenced by anual variations in water table, soil water deficit and fine root loss caused by Phytophthora cinnamomi. Agricultural and Forest Meteorology 169, 92-99. http://doi.org/10.1016/j.agr formet.2012.09.017

Costa, M.; Morla, C.; Sainz, H.; 1997. Los bosques ibéricos. Una interpretación geobotánica. Editorial Planeta. España.

Desprez-Loustau, M.L.; Marçais, B.; Nageleisen, L.M.; Piou, D.; Vannini, A; 2006. Interactive effects of drought and pathogens in forest trees. Annals of Forest Science 63, 597-612. http://doi.org/10.1051/forest:2006040

Erwin, D.C.; Ribeiro, O.K.; 1996. Phytophthora diseases worldwide. APS Press. USA.

Fernández, P.; Carbonero, M.D.; Sánchez, M.E.; Trapero, A.; 2008. ¿Cómo manejar las explotaciones afectadas por decaimiento forestal? Europacork 36, 40-46.

Fernández-Escobar, R.; Gallego, F.J.; Benlloch, M.; Membrillo, J.; Infante, J.; Pérez de Algaba, A.; 1999. Treatment of oak decline using pressurized injection capsules of antifungal materials. European Journal of Forest Pathology 29, 29-38. https://doi.org/10.1046/j.14390329.1999.00127.x

Garbelotto, M.; Schmidt, D.J.; Harnik, T.Y.; 2007. Phosphite injections and bark application of phosphite+ Pentrabark ${ }^{\mathrm{TM}}$ control sudden oak death in coast live oak. Arboriculture and Urban Forestry 33, 309-317.

Garbelotto, M.; Harnik, T.Y.; Schmidt, D.J.; 2009. Efficacy of phosphonic acid, metalaxyl-M and copper hydroxide against Phytophthora ramorum in vitro and in planta. Plant Pathology 58, 111-119. https://doi.org/10.1111/j.1365-3059.2008.01894.x

Hardam, A.R.; 2005. Pathogen profile. Phytophthora cinnamomi. Molecular Plant Pathology 6, 589-604. http://doi.org/10.1111/mmp.12568

Hardy, G.E.S.; Barret, S.; Shearer, B.L.; 2001. The future of phosphite as a fungicide to control the soilborne plant pathogen Phytophthora cinnamomi in natural ecosystems. Australasian Plant Pathology 30, 133-139. http://doi.org/10.1071/AP01012

Jackson, T.J.; Burgess, T.; Colquhoun, I.; Hardy, St.G.E.J.; 2000. Action of the fungicide phosphite on Eucalyptus marginata inoculated with Phytophthora cinnamomi. Plant Pathology 49, 147-154. https://doi.org/10.1046/j.1365-3059.2000.00422.x

Liebhold, A.M.; Brockerhoff, E.G.; Kalisz, S.; Núñez, M.A.; Wardle D.A.; Wingfield M.J.; 2017. Biological invasions in forest ecosystems. Biological Invasions 19, 3437-3458. http://doi.org/10.1007/s10530-017-1458-5

Lowe, S.; Browne, M.; Boudjelas, S.; De Poorter, M.; 2000. 100 of the world's worst invasive alien species: A selection from the global invasive species database, The Invasive Species Specialist Group (ISSG), a specialist group of the Species Survival Commission (SSC) of the World Conservation Union (IUCN). University of Auckland, New Zealand.

Ríos, P.; Obregón, S.; González, M.; de Haro, A.; Sánchez, M.E.; 2016. Screening brassicaceous plants as biofumigants for management of Phytophthora cinnamomi oak disease. Forest Pathology 46, 652-659. http://doi.org/10.1111/efp.12287

Romero, M.A.; Sánchez, J.E.; Jiménez, J.J.; Belbahri, L.; Trapero, A.; Lefort, F.; Sánchez, M.E.; 2007. New Pythium taxa causing root rot on Mediterranean Quercus species in south-west Spain and Portugal. Journal of Phytopathology 155, 288-295. https://doi.org/10.1111/ j.1439-0434.2007.01230.x 
Sánchez, M.E.; Caetano, P.; Ferraz, J.; Trapero, A.; 2002. Phytophthora disease of Quercusilex in south-western Spain. Forest Pathology 32, 5-8. http://doi.org/10.1046/j.1439-0329. 2002.00261.x

Sánchez, M.E.; Caetano, P.; Romero, M.A.; Navarro, R.M.; Trapero, A.; 2006. Phytophthora root rot as the main factor in oak decline in southern Spain. En: Progress in research on Phytophthora Diseases of Forest Trees. Forest Research, Farnham, UK. pp. 149-154.

Sena, K.; Crocker, E.; Vincelli, P.; Barton, C.; 2018. Phytophthora cinnamomi as a driver of forest change: Implications for conservation and management. Forest ecology and management 409, 799-807. http://doi.org/10.1016/j.foreco.2017.12.022

Serrano, M.S.; Fernández-Rebollo, P.; De Vita, P.; Sánchez, M.E.; 2012a. Susceptibility of common herbaceous crops to Phytophthora cinnamomi and its influence on Quercus root rot in rangelands. European Journal of Plant Pathology 134, 409-414. http://doi.org/10.1007/s 10658-012-9999-Z

Serrano, M.S.; De Vita, P.; Fernández-Rebollo, P.; Sánchez, M.E.; 2012b. Calcium fertilizers induce soil suppressiveness to Phytophthora cinnamomi root rot of Quercus ilex. European Journal of Plant Pathology 132, 271-279. http://doi.org/10.1007/s10658-011-9871-6 


\section{Datos de la tesis}

Premio Universitario de la SECF 2019 a la mejor Tesis Doctoral Directores: María Esperanza Sánchez y María Socorro Serrano Centro: Escuela Técnica Superior de Ingeniería Agronómica y de Montes Universidad: Universidad de Córdoba

Fecha de defensa: diciembre de 2019

\section{Publicaciones derivadas de la Tesis Doctoral}

González, M.; Sánchez, M.E.; 2020. Chemical control of Phytophthora oleae and its potential for disease management in olive orchards and natural forests. European Journal of Plant Pathology 157, 211-214.

González, M.; Romero, M.A.; Ventura, L.V.; Gómez-Aparicio, A.; Serrano, M.S.; 2020 Unravelling the role of drought as predisposing factor for Quercus suber decline caused by Phytophthora cinnamomi. European Journal of Plant Pathology 156, 1015-1021.

González, M.; Romero, M.A.; Serrano, M.S.; Sánchez, M.E.; 2020. Fosetyl-aluminium injections controls root rot disease affecting Quercus suber in southern Spain. European Journal of Plant Pathology 156, 101-109.

González, M.; Pérez-Sierra, A.; Sánchez, M.E.; 2019. Phytophthora oleae, a new root pathogen of wild olives. Plant Pathology 68, 901-909.

González, M.; Caetano, P.; Sánchez ME.; 2017. Testing systemic fungicides for control of Phytophthora oak root disease. Forest Pathology 47, e12343.

González, M.; Pérez-Sierra, A.; Serrano, M.S.; Sánchez, M.E.; 2017. Two Phytophtho$r a$ species causing decline of wild olive (Olea europaea subsp. europaea var. sylvestris). Plant Pathology 66, 941-948.

González, M.; Serrano, M.S.; Sánchez, M.E.; 2016. First report of Pythium spiculum causing root rot on wild-olive in Spain. Plant Disease 100, 1023. 